Cahiers de recherches médiévales

Journal of medieval studies

2| 1996

Regards sur le Moyen Âge

\title{
Robert le diable : une « œuvre ouverte »
}

\section{Élisabeth Gaucher}

\section{(2) OpenEdition}

\section{Journals}

Édition électronique

URL : https://journals.openedition.org/crm/2483

DOI : $10.4000 / \mathrm{crm} .2483$

ISSN : 1955-2424

Éditeur

Honoré Champion

Édition imprimée

Date de publication : 12 décembre 1996

Pagination : 61-71

ISSN : 1272-9752

\section{Référence électronique}

Élisabeth Gaucher, «Robert le diable: une « œuvre ouverte » », Cahiers de recherches médiévales [En ligne], 2 | 1996, mis en ligne le 04 février 2008, consulté le 15 décembre 2022. URL : http:// journals.openedition.org/crm/2483; DOI : https://doi.org/10.4000/crm.2483 


\section{等M}

\section{Robert le diable : une « æuvre ouverte*}

La naissance de l'imprimerie, puis l'apparition d'éditions à bon marché, qui ont permis une diffusion massive des romans de chevalerie, cö̈ncident avec l'abandon, dans les milieux lettrés, de ce type de récit au profit des ouvrages humanistes. Se constitue alors un clivage entre une culture d'élite, nourrie d'Antiquité gréco-latine, et une culture populaire, encore attachée à la littérature chevaleresque ${ }^{1}$.

Ainsi s'explique la dérive sociale de Robert le diable au fil de ses éditions successives. Ecrit au XIII siècle, le roman s'adresse d'abord à l'aristocratie aisée, comme en témoigne le soin apporté à la confection des manuscrits et des premiers incunables. Mais au début du XVII siècle, il quitte la haute sphère des imprimeurs à privilège et des libraires, pour entrer dans la Bibliothèque bleue et pénétrer dans les milieux populaires sous la forme de courts livrets grossièrement façonnés ${ }^{2}$. I lui faudra attendre la seconde moitié du XVIII siècle pour en sortir, grâce à Jean de Castilhon, journaliste et érudit, qui fit paraitre une édition "entièrement refondue et considérablement augmentée ».

Résumons le récit, tel qu'on peut le lire dans la version du Xmi siècle et dans la réédition de Castilhon:

- Le duc et la duchesse de Normandie ne parviennent pas à avoir d'enfant.

XIII' s. : la duchesse invoque le diable, qui exauce sa prière : elle est enceinte du duc.

$\mathrm{XVII}^{e} \mathrm{~s}$. : la duchesse Mathilde consulte un juif versé dans les mystères de la cabale et tombe sous le charme d'un génie qui abuse d'elle.

- Robert se révèle vite un enfant difficile. Banni par son père, il se retire dans la forêt et devient le chef d'une bande de brigands.

- Lorsque sa mère lui avoue le secret de sa naissance, il part à Rome se confesser au pape. Celui-ci l'adresse à un ermite, qui lui dicte sa pénitence: feindre la folie, garder le silence et disputer sa nourriture aux chiens.

$\mathrm{XVII}^{e} \mathrm{~s}$.: en chemin, Robert a une altercation avec un chanteur d'opéra; il partage ensuite les déboires d'un couple de jeunes mariés (Cécile et Silvio) persécutés par une bellemère jalouse.

- De retour à Rome, Robert se réfugie à la cour de l'empereur, dont il devient le fou ; il partage sa nourriture avec un chien et dort à ses côtés. Les Sarrasins envahissent l'Italie, favorisés par la trahison d'un prétendant à qui l'empereur a refusé la main de sa fille. Une apparition miraculeuse apporte à Robert une armure qui lui permet, à trois reprises, de

\footnotetext{
${ }^{1}$ H.-J.Martin, «Culture écrite et culture orale, culture savante et culture populaire dans la France d'ancien régime », Joumal des Sarants, 1975, p. 235.

${ }^{2}$ Sur la Bibliothèque bleue, voir G. Bollème, La Bibliothèque bleue : la littérature populaire en France du Xvf au XXX siècle, Paris, Julliard, 1971 (archives). La comparaison entre le texte original de Robert le diable et les livrets bleus a été faite par Lise Andriès, «La Bibliothèque bleue : les réécritures de Robert le diable ", Littérature, 30, mai 1978, pp.51-66.
} 
participer incognito aux combats et d'assurer la victoire des Romains. La princesse, muette de naissance, découvre le secret.

$X V{ }^{e} s$. : naissance d'une idylle entre la princesse Cynthia et Robert.

- La pénitence accomplie dans les délais fixés, l'identité de Robert est révélée.

XIII $\mathrm{s}$. : le héros choisit de mener une vie ascétique avec l'ermite, et meurt en odeur de sainteté.

$X V{ }^{e}{ }^{e}$ s. : il épouse Cynthia, retourne en Normandie, délivre sa mère (devenue veuve) de la tyrannie d'un usurpateur, anéantit le mauvais génie et engendre un fils, qui s'appellera Richard sans peur.

Fondée sur la comparaison de ces deux versions, l'étude a pour but de mesurer l'influence d'un nouveau public sur l'écriture du roman et d'examiner les variantes qui affectent plus précisément les deux thèmes majeurs du diable et de la pénitence.

\section{L'édition de Castilhon a été accompagnée d'une préface qui la justifie:}

Il paraitra sans doute bien singulier qu'on ait pris la peine de rajeumir des ouvrages, qui, depuis plus de deux siècles, sont abandonnés au Peuple; des Romans que la plus mince Bourgeoise n'oserait se vanter d'avoir lus, non pas à cause du style et du langage, qui les lui rendent aussi inintelligibles que le jargon alambiqué de Marianne l'est quelque-fois pour nos PetitesMaîtresses, mais précisément parce qu'ils ont fait l'amusement de la plus vile Populace, qui commence à ne plus les entendre... ${ }^{3}$.

Suit une anecdote dans laquelle un homme de lettres, s'adressant à une femme de la noblesse, dénigre un roman à succès portant le titre de "philosophique », blâmable parce qu'il met « l'esprit à la place du sentiment » et inférieur, sur le plan de l'« imagination », à la littérature de colportage. Choquée par la comparaison, Madame de *** envoie sa femme de chambre chercher «à la cuisine» un volume de la Bibliothèque bleue. Le critique s'en empare et travaille à le « rajeunir » afin de le « rendre digne de toutes sortes de lecteurs (...), en y ajoutant des situations et des épisodes nouveaux ».

Cette anecdote illustre un mouvement de réaction qui s'élève, à la fin du Xvuri siècle, contre l'esprit des Lumières. La littérature populaire devient un objet d'étude, et retient tout particulièrement l'attention de l'aristocratie inquiétée par le péril urbain : le Moyen Age et le peuple, respectivement identifiés à la pureté originelle des campagnes et de l'enfance, alimentent le mythe du bon sauvage, face à la ville corruptrice où se fondent dangereusement les hiérarchies traditionnelles. Ainsi réhabilité, le roman de chevalerie fait figure de «fils prodigue » et se pare des « attraits de l'exotisme ${ }^{4}$. La Bibliothèque bleue quitte les cuisines du peuple pour entrer dans le musée des notables. Attirés par le folklore naif, ceux-ci n'en continuent pas moins à afficher leur mépris pour la « vile populace». Un siècle plus tôt, les

\footnotetext{
${ }^{3}$ Paris, Bibliothèque nationale $\mathrm{Y}^{2} 6196$. Le volume comprend la réédition de trois titres de la Bibliothèque bleue : L'Histoire de Pierre de Provence et de la belle Maguelonne (1776), l'Histoire de Robert le Diable, duc de Nomandie (1783) et l'Histoire de Richand sans Peur, duc de Normandie, fils de Robert le Diable (1783). Nous citons la version originale de Robert d'après l'édition d'E. Löseth (Paris, Firmin Didot, 1903), et son remaniement de 1783 d'après l'édition publiée à Paris chez Garnier, s.d.

${ }^{4}$ M. de Certeau, La Culture au pluriel, Paris, Christian Bourgois, 1980 (1 ${ }^{\text {oc }}$ éd. U.G.E., 1974), p. 58.
} 
mêmes sentiments contradictoires animaient l'entreprise de Charles Perrault ${ }^{5}$ : ce grand bourgeois, commis de Colbert et partisan de l'absolutisme, n'éprouvait guère de sympathie pour le peuple, qu'il assimilait à un enfant devant être éduqué et contrôlé par des contes rédigés à son usage.

La réécriture de Robert le diable dans l'édition de Castilhon reflète cet état d'esprit. Le remanieur observe, avec le regard d'un ethnologue, la civilisation médiévale dont il prétend éclairer les usages par des commentaires pédagogiques. Il s'attarde, par exemple, sur la pratique des pèlerinages, «alors d'autant plus à la mode que cette dévotion était encore dans sa nouveauté » (p. 6), ou sur des coutumes qui, sans être nommées, n'ont plus de secret pour les médiévistes actuels. Ainsi, le vœu prononcé à l'issue d'un tournoi par un chevalier vaincu se trouve qualifié de « loi bizarre» (p. 8), alors qu'il s'agit sans doute d'une emprise. De même, si le gigantesque pâté d'où sort le mauvais génie (métamorphosé en nain) nous fait penser aux « entremets » des banquets de cour à la fin du Moyen Age, le remanieur n'y voit qu'une occasion d'allécher son lecteur par une recette de cuisine exotique :

ces pâtés, qui étaient fort à la mode dans ce temps-là, étaient composés de foies d'alcyons, de langues de colibri, et de truffes vertes des Indes (p. 108).

Epoque étrange que «ce temps-là », où les Arabes innovaient dans les arts (p. 12) et où l'on parlait une langue bien différente de la nôtre (p. 55)... De la surprise à l'incompréhension, le pas est vite franchi. L'emblème de la massue, qui caractérisait le fou médiéval, devient une arme noble au même titre que l'épée, puisque Robert y recourt en toute lucidité pour combattre les Sarrasins ( $p$ 84). Certaines expressions du Xư' siècle donnent lieu à des erreurs de traduction: là où le texte original dépeint la rapidité du cheval de Robert, "qui tost se remue» (v. 3225) sous l'impulsion de son maitre lancé à la poursuite des Infidèles, le remanieur, ignorant le sens médiéval de «remuer», attribue à l'animal un comportement inattendu :

car, dans le temps que l'épée de Robert abattait la tête de l'un, son cheval, avec ses dents, arrachait l'épaule d'un second, et d'un coup de pied enfonçait les côtes d'un troisième (pp. 89-90).

Enfin, le texte remanié pèche par anachronisme. Nombreux sont les lapsus révélateurs de sa modemité. Le motif des “males enfances » de Robert sert de prétexte à une condamnation de la « débauche » et du « libertinage » (p. 33) ; au Dieu des chrétiens se substitue l'Etre suprême des philosophes; et l'on n'hésite pas à mettre le héros (en chemin vers l'Italie, il est vrai) aux prises avec un chanteur d'opéra... Quant au recours à l'onomastique, dont les sonorités n'ont plus rien de médiéval, il vise tout autant à particulariser des types intemporels qu'à les adapter au monde du public. Celui-ci devient, en dernier ressort, "censeur, juge souverain et auteur du livre »: comme tous les romans de la Bibliothèque bleue, Robert le diable est une «histoire sans auteur", donc une «histoire qui se raconte avec les mots du lecteur ${ }^{6}$.

Mais c'est aussi un récit exemplaire. En tant que tel, il ne peut fonctionner que s'il tient compte, dans sa topique, des images déjà ancrées dans l'esprit du destinataire: à toutes les

\footnotetext{
${ }^{5}$ M. Soriano, Les contes de Perrault : culture savante et traditions populaires, Paris, Gallimard, 1977 $(T e l)\left(1^{j e}\right.$ éd. 1968) : préface et $v^{\varepsilon}$ partie.

${ }^{6} \mathrm{G}$. Bollème, op. cit, p. 22.
} 


\section{Elisabeth GAUCHER}

époques, l'exemplum se définit comme un récit métaphorique, qui s'adresse aux mentalités de son public afiı de faciliter la compréhension d'une vérité abstraite. Aussi les versions remaniées de Robert le diable sontelles le miroir de leur temps. Celle qui nous intéresse ici véhicule un didactisme à la fois moral et social, où se devinent les préoccupations de l'âge classique. Elle accuse tout d'abord une tendance prêcheuse propre à toute la littérature populaire du XVII" siècle, fruit d'une étroite collaboration entre colporteurs et missionnaires lors de la Contre-Réforme : face aux protestants, l'Eglise cherche un nouvel enracinement dans les traditions auxquelles sont restées fidèles les masses catholiques? ${ }^{7}$. L'édition de Castilhon renferme des commentaires sur les bienfaits de la religion rédemptrice, qui semblent répondre aux doctrines calvinistes et jansénistes sur la grâce et la prédestination :

Que la religion a d'empire sur l'esprit de l'homme! par elle, le tigre le plus sanguinaire devient un agneau paisibie... (p. 69).

Mais le récit diffuse aussi une morale civique, empreinte d'intolérance et de préjugés sociaux. L'antisémitisme s'y exprime dans sa traditionnelle association avec la trahison, la sorcellerie et l'usure. En témoignent deux juifs qui cultivent des « intelligences secrètes » avec les Sarrasins (p. 6), ou l' ' infâme Iduméen » dont est victime la crédule Mathilde (p. 13), ou encore, à Rome, le financier « fort considéré par ses richesses » et chargé de collecter les revenus de l'Etat (pp. 76-77). Mais il ne s'agit là que de vestiges médiévaux, contemporains des pogroms de la Grande Peste et des tribunaux de l'Inquisition (le personnage du juif receveur d'impôts figure déjà dans un incunable de 1496). En revanche, l'allusion au peuple versatile, «qui juge sur les apparences» (p. 110) et retire sa confiance à la duchesse victime d'un usurpateur, pourrait bien traduire la crainte des classes dominantes à la veille de la Révolution.

Cette socialisation du récit s'accompagne, bien sûr, de modifications idéologiques. L'aspect chevaleresque et féodal est évacué. Le récit des combats n'intéressant plus le public modeme, on n'en répète plus les phases avec autant de détails que dans le texte-source. Il en va de même pour les comparaisons épiques, remplacées par des images empruntées à la vie agricole: si Robert, au XIII siècle, poursuivait les Turcs avec l'achamement d'un lion (v.3360) ou d'un loup affamé (v. 3216), on le voit désormais décapiter ses adversaires avec la tranquille assurance d'un «moissonneur [qui] fait tomber les épis ou l'herbe des prés» (p. 90) ${ }^{8}$. D'autre part, l'idéologie camavalesque, qui s'était introduite au XIV siècle dans le Dit de Robert le diable par le biais de trois séquences bouffonnes (Robert fait baiser le cul de son chien à un juif ; il envoie une reine dans la boue; il jette un chat dans une marmite), disparait sous l'effet d'une censure pudibonde : au rire du corps dénudé se substituent celui du travestissement et, surtout, une forme de comique psychologique illustrée par la scène entre Robert et le chanteur d'opéra ou l'épisode sentimental de Cécile et Silvio, dans lesquels la patience du héros est durement mise à l'épreuve. De même, on observe un certain recul de la topique chrétienne. A preuve, la sécularisation du prologue: celui-ci, qui apparait pour la première fois dans le Dit sous la forme d'une invocation au Christ puis, dans les textes imprimés, à la Vierge, devient, au XVII' siècle, une mise en garde contre le «crime (...) insensé » qui pousse

\footnotetext{
${ }^{7}$ H.-J. Martin, art. cit., p. 252.

${ }^{8}$ De même, p. 83 : Robert "parcourt les rangs ennemis, et les rangs entiers disparaissent devant lui, comme les feuilles dont la gelée a desséché les tiges, et qu'un ouragan disperse dans les airs ».
} 
l'homme à «murmurer contre la Providence» (p. 1). Le récit n'est donc plus placé sous le signe de la pénitence, mais sous celui d'une morale pragmatique. Cet effort de laïcisation rejoint parfois un souci de vraisemblance: pour aller de la Normandie à Rome, Robert ne passe plus par les étapes consacrées de Saint-Gilles et Saint-Jacques (v. 486-487) mais par la Savoie et le mont Cenis (p. 56). Le caractère diabolique de Robert tend à s'estomper: le sombre héros du XIII siècle, qui prenait plaisir à profaner les églises, incendier les monastères, violer les nonnes et tuer les moines, a évolué en chenapan « espiègle », occupé à dérober les vêtements de ses ainés ou à voler des fruits dans les jardins (chap. III), puis en personnage de faits divers, chef d'une troupe de bandits, évoquant peut-être, dans l'esprit des lecteurs du XVII ${ }^{e}$ siècle, les hors-la-loi Cartouche, Mandrin et Guilleri. En fin de compte, prédomine l'idéologie bourgeoise, fondée sur l'épargne, le travail et la famille. C'est elle qui impose ses critères à la condamnation des brigands, en qui le narrateur voit moins des suppôts de Satan que des « filous " couverts de "dettes» - « en un mot, tous ceux que leur oisiveté avait jetés dans l'indigence » (p. 33). C'est elle encore qui entraîne, par rapport au textesource, le développernent du motif familial. Au début du récit, le mariage du duc de Normandie, rapidement évoqué dans le roman du XIII siècle, donne lieu à un plus ample développement, assorti d'anecdotes stéréotypées (le choix de l'épouse par portraits interposés, l'aide militaire offerte par le prétendant à son futur beau-père...). La mère du héros change aussi de figure. La version initiale la montre sous un jour pitoyable: Robert la menace de son épée pour la forcer à avouer le secret de sa naissance; condamnée sans appel, elle mourra coupable. Dans le texte remanié, elle recouvre sa dignité maternelle, conjugale et politique: elle jouit de l'affection de son fils (Robert, plein de "tendresse » et de «timidité », tombe «à ses genoux " lors de la scène des aveux, pp. 39-43) ; elle est disculpée de l'accusation d'adultère (étant déjà enceinte du duc, sans le savoir, au moment du viol) et vengée de l'imposture par l'anéantissement final du mauvais génie; enfin, elle est restituée dans ses droits sur le duché de Normandie. Ce demier point, d'ailleurs, recoupe une autre préoccupation familiale: l'héritage. Dans le récit médiéval, Robert, obsédé par un impératif spirituel, ne songe qu'à sa pénitence et refuse, une fois celle-ci accomplie, de récupérer le duché de Normandie que lui ont laissé ses parents défunts, et de ceindre la couronne que lui propose l'empereur de Rome: il choisit de gagner son Paradis par une vie érémitique. Ce dénouement ascétique est remplacé, à partir du Dit, par une fin mondaine: le héros, tombé amoureux de la princesse, devient à son tour bon époux, bon prince et bon père - exact récapitulatif de ces vertus bourgeoises où les lecteurs du XVứ siècle pouvaient reconnaitre les principes de Rousseau et Diderot.

Ainsi, le regard distancié suggéré dans la préface de l'édition de Castilhon, trace un "horizon d'attente" qui détermine l'écriture du roman. Reste à voir comment cette " esthétique de la réception ", dont on a jusqu'ici relevé les effets à travers des motifs ponctuels, a altéré le message fondateur du récit qu'expriment les thèmes du diable et de la pénitence.

Du XIII au XVIII siècle, la croyance au Prince des Ténèbres cesse d'être une évidence. Pourtant, le siècle des Lumières ne parvient pas à s'en affranchir totalement ${ }^{\circ}$. Si les philoso-

\footnotetext{
${ }^{9} \mathrm{M}$. Milner, Le diable dans la littérature fransaise de Cazotte à Baudelaire (1772-1861), 2 vol., Paris, José Corti, 1960, chap. $I^{*}$ : « De la croyance au Prince des Ténèbres dans le siècle des Lumières ».
} 


\section{Elisabeth GAUCHER}

phes et les médecins condamnent la superstition populaire, de nombreux ouvrages, avalisés par l'Eglise, continuent à affirmer l'existence du Malin, comme un "défi absurde et cruel " lancé à "l'optimisme fondamental d'une époque tout entière tendue vers le bonheur " ${ }^{10}$. Ainsi l'Histoire critique des pratiques superstitieuses qui ont séduit les peuples et embarrassé les sçavants, publieée entre 1732 et 1737 par le Père Pierre Lebrun, préconise un juste milieu entre la crédulité excessive et l'incrédulité radicale ${ }^{11}$.

La même hésitation ressort du roman de Robert le diable proposé aux lecteurs du Xvin siècle. Le narrateur y déplore les "voies ridicules de la superstition la plus absurde" où s'engage la duchesse de Normandie après avoir en vain cherché dans la médecine et la chimie une solution au drame de sa stérilité. Mais l'intensité de son malheur excuse la faiblesse de son esprit :

Mathilde n'était point crédule, mais quel est l'esprit fort qu'un grand intérêt n'ébranle pas quelque fois? (p. 12).

L'erreur de la duchesse ne consiste plus, comme au XIIl' siècle, à se livrer au diable, mais à consulter un juif thaumaturge.

Or ce demier permet au remanieur d'opérer un transfert de la crainte religieuse au plaisir esthétique. En effet, il écrit à une époque où

ce que le diable perd dans le domaine de la croyance. il le gagne dans celui de l'imagination; ce qu'il perd sur le plan religieux et dogmatique, il le gagne sur le plan esthétique ${ }^{12}$.

La sobriété du récit médiéval, où le diable n'intervient pas directement, ne répond plus à la sensibilité d'un public en quête de rêve et de divertissement. Dans l'anecdote qui ouvre l'édition de Castilhon, Madame de *** ne choisit-elle pas ses lectures avec l'« espoir de s'en amuser »? Rajeuni, le roman de Robert le diable devient spectacle, et annonce la mise en scène romantique qu'en offrira, en 1831, l'opéra de Meyerbeer. On voit, par exemple, l' « impure » caverne des brigands, soudain enveloppée d'un épais nuage, s'embraser sous le coup de la foudre divine (pp. 50-51). Mais surtout, le récit se colore d'effets spéciaux plus proches des Mille et une Nuits que de l'exemplum médiéval : au miracle chrétien, on préfère désormais le merveilleux féerique. Génies, fée, esprits élémentaires, talisman et métamorphoses entourent la destinée du héros. On apprend, à la fin, que Robert n'est pas le fils du diable (il ne doit plus ce sumom qu'à sa méchanceté initiale), mais la victime d'un mauvais génie qui, sur l'ordre d'une fée, s'est emparé de son esprit à sa naissance (pp. 116-118). Si la venue du héros au monde s'accompagne encore des "prodiges les plus inouis », le narrateur les impute non plus à des causes surnaturelles, " comme plusieurs historiens l'ont cru ", mais aux pouvoirs magiques dont le juif consulté par Mathilde détient les «secrets» (p. 19).

L'intervention du génie ne s'explique pas seulement par la recherche du dépaysement poétique; elle offre aussi une traduction métaphorique des problèmes du mal et de la responsabilité ${ }^{13}$. Persécuté par la «malice» du «fils de Tubal», Robert a pu résister grâce à un "génie protecteur " qui l'a assisté dans ses épreuves (p. 118). Ces bons et ces mauvais génies

\footnotetext{
${ }^{10}$ Ibid., p. 38.

${ }^{11}$ Ibid., p. 56.

${ }^{12}$ Ibid., p. 68.

${ }^{13}$ Cette tendance s'affirme dans le demier quart du XvmI siècle (ibid., p. 126).
} 
n'ont pas le pouvoir tentateur de Satan: ils ne cherchent pas à contraindre leurs victimes à choisir entre le bien et le mal (telle est, dans le roman du XII ${ }^{e}$ siècle, la signification du pacte satanique conclu sous l'effet de la colère par la duchesse de Normandie), mais se contentent d'exercer sur elles une action extérieure. Toutefois, cette dramatisation des mécanismes de la subjectivité humaine n'exclut pas une peinture psychologique des sentiments. La "sensibilité » de Mathilde est à l'origine de "son crime et de ses malheurs" (p. 11). Affligés par les débordements de leur fils, le duc et la duchesse s'interrogent tour à tour sur leur responsabilité:

"Grand Dieu! disait-il, qui me l'avez donné dans votre colère, pour me punir des murmures que je formais contre votre sagesse, que votre vengeance ne tombe que sur moi! » (p. 30).

La duchesse ne cessait de gémir, et de se reprocher d'être la cause du dérèglement de son fils; mais plus elle s'examinait, et moins elle pouvait se reconnaitre coupable (p. 43).

Ainsi les mystères de la démonologie invitent à l'introspection et à l'auto-confession.

Lié à ces préoccupations métaphysiques, le thème de la pénitence subit, lui aussi, une réactualisation par rapport à son traitement original. Si les impératifs dictés au héros restent inchangés, trois modifications peuvent être relevées dans les modalités de leur application.

C'est d'abord l'insistance rousseauiste accordée à la nature humaine et à la question de la perfectibilité. Dans le roman du XIII siècle, les effets de la conversion de Robert au bien se font immédiatement ressentir. Dans la version du Xvul siècle, le héros ne se départit pas aussi facilement de son tempérament violent ; cédant à son impatience naturelle, il s'emporte dans des rixes contre un chanteur d'opéra (p. 59) ou contre un « discourtois chevalier» (p. 61) :

[Robert] avait des vertus à acquérir; mais son naturel impétueux était un obstacle qu'il fallait surmonter (...). Ce maudit orgueil tracassait le bon Rober : il résolut d'acquérir de la modestie à quelque prix que ce fût (pp. 56-60).

Lorsque l'ermite lui dicte les conditions de son rachat, et notamment l'interdiction de nuire à quiconque lui causerait du tort, Robert accepte à contrecœur cette clause qui équivaut, selon lui, à « renoncer à toute sensibilité » (p. 68). A la notion de grâce divine se substitue celle d'accoutumance :

Robert se modérait en tout: son caractère n'était pas changé, parce que le caractère ne peut l'être; mais comme le fond en était bon, l'habitude de la modération qu'il acquérait peu à peu le rendit excellent (p. 73).

La deuxième innovation conceme le motif du chien. Dans la tradition hagiographique, il sert à la fois à souligner l'abjection consentie par le pénitent et à aider celui-ci à satisfaire ses besoins primaires (de la viande pour manger, de la chaleur pour dormir). Dans le Dit du XIV siècle, on a vu qu'il permettait d'introduire la scène du cul baisé, jugée subversive et donc supprimée par la suite. L'édition du XVur siècle renferme d'autres types de surenchère. Au motif alimentaire (Robert disputant sa nourriture au chien, lequel, dès la version du XIII siècle, finit par sympathiser avec lui et par lui donner lui-même à manger), s'ajoute celui de l'assistance médicale : blessé au visage lors d'un combat contre les Sarrasins, le pénitent est soigné par l'animal, qui cicatrise la plaie en la léchant (p. 85). La fin du récit reprend au 
texte-source l'épisode de la blessure à la jambe, où Robert a reçu par erreur le fer d'une lance. Ainsi décrit, le héros nous semble évoquer la figure de saint Roch, dont le culte, déjà répandu au XIV siècle, restait encore vivace à l'époque classique ${ }^{14}$. Leurs carrières respectives, d'ailleurs, présentent des points communs : pèlerinage à Rome, assistance aux malheureux (l'un soigne les pestiférés, l'autre aide les chrétiens à repousser les Turcs), sacrifice de soi (saint Roch est touché par la peste, et Robert blessé au combat), incompréhension des témoins (saint Roch est jeté en prison, soupsonné, comme Robert à la cour de l'empereur, d'être un espion), aura surnaturelle (le cachot du saint brille d'une étrange lueur, semblable à l'éclatante nuée qui apparait à Robert pour l'inciter à se rendre sur le champ de bataille)... Mais surtout, l'abondante iconographie consacrée au saint guérisseur ${ }^{15}$ suggère des rapprochements avec le héros du roman. Les deux personnages partagent la même beauté: saint Roch est mort à trente-deux ans, tandis que la « taille» et les «traits» de Robert suscitent l'admiration des Romains (p. 70). Tous deux portent le vêtement d'un pèlerin: ce costume amène souvent à associer saint Roch à saint Jacques, et apparait comme une nouveauté dans notre récit par rapport à la version du XI" 'èècle, où Robert endossait l'habit d'un ermite pour accomplir sa pénitence. Enfin, un chien (un roquet...) portant un pain dans sa gueule est traditionnellement représenté à côté de saint Roch, lequel dénude une de ses jambes pour y montrer une profonde blessure: si celle-ci tend à évoquer la peste, assimilée, dans l'imagination populaire, à un trait lancé de la main de Dieu, on sait qu'une vie de saint Roch, ajoutée après coup à la Légende dorée, dit qu'il eut la cuisse percée d'une flèche. La ressemblance est flagrante avec Robert, qui partage son pain et sa souffrance avec son fidèle chien, devenu pour lui l'unique moyen d'assurer sa survie. Outre ces coïncidences avec la piété populaire de l'époque, un autre effet de surenchère caractérise le chien dans l'édition de Castilhon. L'animal y devient un personnage à part entière: il dénonce, par ses aboiements, la trahison du nain, qui a emprunté les traits de Robert pour séduire la princesse Cynthia (p. 114). Ce motif du chien dévoué à son maître, et que les circonstances transforment en acteur judiciaire, pourrait bien être une concession à l'épopée médiévale, et notamment à la chanson de Macaire ${ }^{16}$.

Reste à examiner le troisième motif pénitentiel : la folie. Le récit moderne nettoie la version du Xü siècle des vieux topoï jugés éculés ${ }^{17}$. Le motif de la tonsure disparaît dès le Dit du XIV siècle. L'emblème de la massue n'est plus compris dans sa signification originelle: il ne s'agit plus de l'arme défensive qu'utilisait le fou médiéval pour répondre à ses persécu-

\footnotetext{
${ }^{14}$ Sur saint Roch, voir E. Mâle, L'art religieux à la fin du Moyen Age en France, Paris, Armand Colin,

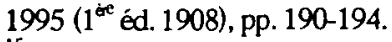

${ }^{15}$ On relève, en particulier, la présence de saint Roch à Troyes, haut-lieu de la Bibliothòque bleue : une statue dans la chapelle Saint-Gilles et un vitrail (daté de 1525) à Saint-Léger-lez-Troyes.

${ }^{16} \mathrm{Cf}$. J. Subrénat, "Un héros épique atypique : le chien d'Auberi dans Macaire », Mélanges H-E. Keller, Kalamazoo (Michigan), 1993, pp. 81-96.

${ }^{17}$ Sur ces topoï, voir Ph. Ménard, "Les emblèmes de la folie dans la littérature et dans l'art (XIr'XIII s.) », "Fanai chansoneta novele *. Hommage à J.-Ch. Payen. Essai sur la liberté créatrice du Moyen Age, Publications de l'Université de Caen, 1989, pp. 253-265. Sur la folie dans Robert le diable, voir J. Berlioz, «Métaphore, lieux communs et récit exemplaire : les images de la folie simulée dans la Vie du terrible Robert le Dyable (1496), Symboles de la Renaissance. Arts et langage, t. 2, Paris, 1982, pp. 89-108 et pp.231-236 ; J.-M. Fritz, Le discours du fou au Moyen Age, Paris, P.U.F., 1992 (Perspectives littéraires) ; M. Laharie, La folie au Moyen Age (Xf́-Xسf s.), Paris, Le Lópard d'Or, 1991.
} 
teurs, mais d'une arme offensive à laquelle ne dédaigne pas de recourir le chevalier pour combattre les Sarrasins. Les attributs spécifiques aux fous de Dieu ne sont pas davantage respectés. Ainsi l'escalier sous lequel dort le héros dans le roman du Xnl siècle, et qui provient d'un emprunt aux textes hagiographiques (Vie de saint Alexis...), ne figure plus dans l'édition de Castilhon, où seule est mentionnée la "loge " du chien. Si le topos du peuple persécuteur résiste mieux, il ne donne plus lieu, comme au xun siècle, à une scène lynchage unanime (v. 918-956) mais à un tableau plus nuancé : seuls les enfants lapident le fou,

les honnêtes gens se contentaient de le plaindre, et le défendaient contre tous ceux qui l'attaquaient. Les uns s'amusaient de sa folie, les autres en avaient pitié (p. 69).

Quant au motif corrélatif du souverain protecteur, il n'obéit plus aux mêmes motivations. Au $\mathrm{XIII}^{\mathrm{e}}$ siècle, l'empereur ne protège Robert que parce qu'il apprécie ses plaisanteries; au XVII siècle, la compassion se mêle à la recherche égoïste du plaisir :

«Voilà, dit-il, le plus bel homme que j'aie jamais vu; il parait avoir pendu l'esprit, et certes, c'est bien dommage; il m'intéresse, je défends qu'on lui fasse aucun mal, je veux qu'on en ait soin, qu'on le serve, qu'on ait pour lui toute sorte d'égards» (p. 70).

Se pose, de plus en plus nettement, la question éthique : a-t-on le droit de rire d'un fou, de faire d'un malheureux un bouffon? De fait, le texte n'insiste plus autant sur les folies de Robert, que la version du XII' siècle tendait à banaliser par le procédé de la répétition ; en revanche, il souligne la diversité des réactions qu'elles provoquent, suscitant le rire chez les uns, la pitié chez les autres.

Car Robert n'est pas seulement fou de Dieu, mais aussi fou du roi. Il occupe par là même une position ambiguë, entre la marginalisation et la domestication. La «politisation de la folie ${ }^{18}$, liée à l'essor des cours au Moyen Age, a tout particulièrement retenu l'attention des lecteurs modernes. Pour eux, elle ne consiste plus seulement à distraire l'empereur, mais à l'assister dans l'exercice de ses fonctions, voire à recueillir ses confidences :

«il faut bien [dit l'empereur à Robert] que tu m'aies inspiré une grande confiance, puisque, malgré ton état, je t'ai dévoilé des secrets d'une aussi grande importance" (p. 76).

La liberté d'expression, caractéristique du fou médiéval, subit une récupération politique qui l'associe au motif du bon conseiller. Robert sélectionne avec discemement les requêtes qu'on le charge de présenter à l'empereur, et dénonce la conspiration du traitre Osorio. A l'ascèse religieuse, il ajoute donc des travaux d'utilité publique que n'envisageait pas le texte original. Faut-il y voir un « usage politique » du roman propre au XVII é siècle, où l'ancienne noblesse, encore fraîchement compromise par la Fronde et concurrencée par la promotion des roturiers, aurait réagi en valorisant son "rôle naturel de conseiller du prince " ${ }^{19}$ ? On ne saurait cependant oublier que les romans du XII ${ }^{e}$ siècle (tel Protheselaus de Hue de Rotelande) attestent déjà ce personnage du fou conseiller du roi. Toujours est-il que la version moderne de Robert le diable accuse une nette tendance à réhabiliter le héros au nom d'une taxinomie sinon aristocratique, du moins bourgeoise. Il n'est plus question, en effet,

\footnotetext{
${ }^{18}$ Sur cette notion, et son traitement dans la version originale de Robert, voir M. Laharie, op. cit, p. 276.

${ }^{19} \mathrm{Ch}$. Almavi, Le goût du Moyen Age, Paris, Plon, 1996, p. 185.
} 


\section{Elisabeth GAUCHER}

d'assimiler, comme au Moyen Age, folie (même simulée) et gaspillage ${ }^{20}$. A la cour de Rome, Robert exerce sa largesse

avec un tel discemement qu'il n'y avait que les vrais pauvres qui eussent part à ses aumônes, repoussant les paresseux et les vagabonds, et leur faisant signe d'aller travailler (p. 72).

Enfin, à cette folie «politiquement correcte » se greffe un discours médical, lui aussi révélateur des préoccupations modernes: au concept médiéval de la possession se substitue celui de la maladie mentale. Robert le diable, dans sa version "rajeunie", rend l'écho des réflexions des XVII et XVII' siècles sur l'être humain et citoyen, dans sa fonctionnalité physiologique et administrative $e^{21}$. On sait qu'en 1656, la création de l'Hôpital général par Louis XIV, symbolisant l'" ère du grand renfermement ", a conduit à interner dans un même espace les fous et les autres asociaux, oisifs et chômeurs, afin de protéger le corps social contre tout risque d'émeutes. Ce système, né d'une conception éthique (la condamnation du désœuvrement) et économique (dans un contexte de crise du travail), n'était pas à l'abri des dysfonctionnements. En 1791-1793, le rapport d'un philosophe et médecin parisien révèle que de nombreux "fainéants» et "vagabonds » jouent les maladies nerveuses pour bénéficier des secours publics ${ }^{22}$. Notre roman, dans l'édition de Castilhon, évoque cette conjoncture à travers les propos d'un courtisan jaloux qui, cherchant à discréditer Robert, l'accuse de «contrefaire l'insensé» (p. 73) pour s'introduire à la cour impériale —soupçon étranger au texte du XII' siècle, où tout le monde croyait « sans faillanche " (v. 1354) à l'infirmité du héros. Toutefois, l'« homme méchant », désireux de ménager la sympathie de l'empereur pour son fou, corrige rapidement son propos et suggère de "faire examiner » Robert «par un médecin» (p. 74). Son revirement coïncide avec une conception plus scientifique de la folie, propre au XVII' siècle, où, de maisons de garde, les hôpitaux évoluent en établissements de soins. Et si les insensés inspirent encore la peur, c'est parce que leur mal est perçu comme une menace enracinée dans la raison de tout homme. Une fois sa pénitence accomplie, Robert met en garde ceux qui l'ont humilié lorsqu'ils le croyaient fou :

"que cet exemple vous apprenne à être humains et compatissants envers les fous comme envers les sages: car quelque sages que vous puissiez être, il ne faut qu'une fibre dérangée pour vous rendre plus fous que ne le paraissais » (p. 105).

Désormais, le roman insiste sur l'intermittence de la folie, présentée comme un «mélange d'extravagance et de sagesse » (p. 77). Là encore, le constat reproduit le discours médical de l'époque, selon lequel l'insensé recouvre souvent, mais aussi provisoirement, l'usage de sa raison ${ }^{23}$. On peut aussi songer au Neveu de Rameau. Ne s'offre-t-il pas lui aussi comme « un composé (...) de bon sens et de déraison "? Celui que Diderot dépeint comme l' « un des plus

\footnotetext{
${ }^{20}$ Sur le fou prodigue et dépensier, voir J.-M. Fritz, op. cit, pp. 276-277.

${ }^{21}$ Sur l'historique de la folie pathologique, cf. M. Foucault, Folie et déraison. Histoire de la folie à l'âge

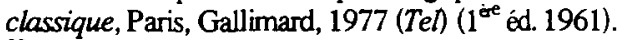

${ }^{22}$ Pierre-Jean-Georges Cabanis, "Quelques principes et quelques vues sur les secours publics. Rapports faits à la Commission des Hôpitaux de Paris", cité dans Enfermer ou guérir. Discours sur la folie à la fin du xuIf siècle, textes choisis et présentés par Claude Wacjman, Publications de l'Université de Saint-Etienne, 1991, p. 85.
}

${ }^{23}$ Ibid., p. 82. 
bizarres personnages de ce pays $n^{24}$ ne peut-il être rapproché de Robert, dont l'empereur ne cesse de souligner la folie « singulière » (p. 71) ? Ce caractère intermittent du mal qui affecte les insensés "rend leur sort plus déplorable " (p. 76) et appelle la pitié. Aussi l'empereur ordonne-t-il de laisser son fou tranquille, afin de ne pas «empirer son état" par des «tracasseries» (p. 77). De nouveau, cette sollicitude rejoint les conseils des médecins du XVIIf siècle, qui interdisaient de maltraiter les insensés dans les asiles ${ }^{25}$.

Robert, continuant à s'accuser, entra dans des détails si singuliers, que les assistants, malgré leur appréhension, s'approchèrent peu à peu de lui, et ne purent s'empêcher de sourire: ils l'interrogèrent, et la naïveté de ses réponses lui concilia l'amitié de toute l'assemblée (p. 61).

La curiosité amusée mais distante des cardinaux romains pour notre héros pourrait bien traduire les sentiments de l'aristocratie du XVII' siècle à l'égard de la littérature populaire. Roman égaré, enfant déchu de la tradition chevaleresque, Robert le diable ne sera récupéré que pour un temps provisoire: il retombera, au siècle suivant, dans les ballots des colporteurs. Est-ce pour avoir abandonné le motif du pacte satanique, que la légende n'a pas su, comme celle de Faust, conserver la faveur du public lettré?

\footnotetext{
${ }^{24}$ Cité par M. Foucault, op. cit. p. 363.

${ }^{25}$ Enfermer ou guérir, op. cit., p. 38.
} 